\title{
A Two-Gene Signature for Tuberculosis Diagnosis in Persons With Advanced HIV
}

\begin{abstract}
Vandana Kulkarni ${ }^{1 \dagger}$, Artur T. L. Queiroz ${ }^{2,3 * \dagger}$, Shashi Sangle ${ }^{1}$, Anju Kagal ${ }^{1}$, Sonali Salvi ${ }^{1}$, Amita Gupta ${ }^{4}$, Jerrold Ellner ${ }^{5}$, Dileep Kadam ${ }^{1}$, Valeria C. Rolla ${ }^{6}$, Bruno B. Andrade ${ }^{2,3+}$, Padmini Salgame ${ }^{5 t}$ and Vidya Mave ${ }^{1,4 * t}$

${ }^{1}$ Byramjee-Jeejeebhoy Government Medical College-Johns Hopkins University Clinical Research Site (BJGMC-JHU CRS), Pune, India, ${ }^{2}$ Instituto Gonçalo Moniz, Fundação Oswaldo Cruz, Salvador, Brazil, ${ }^{3}$ Multinational Organization Network Sponsoring Translational and Epidemiological Research (MONSTER) Initiative, Salvador, Brazil, ${ }^{4}$ Johns Hopkins University School of Medicine, Baltimore, MD, United States, ${ }^{5}$ Rutgers- New Jersey Medical School, Center for Emerging Pathogens, Newark, NJ, United States, ${ }^{6}$ Instituto Nacional de Infectologia Evandro Chagas, Fundação Oswaldo Cruz, Rio de Janeiro, Brazil
\end{abstract}

OPEN ACCESS

Edited by:

Novel N. Chegou,

Stellenbosch University, South Africa

Reviewed by:

Marielle C. Haks,

Leiden University Medical

Center, Netherlands

Jiezuan Yang

Zhejiang University, China

${ }^{*}$ Correspondence:

Vidya Mave

vidyamave@gmail.com

Artur T. L. Queiroz

artur.queiroz@fiocruz.br

tThese authors have contributed equally to this work

Specialty section

This article was submitted to

Microbial Immunology,

a section of the journal

Frontiers in Immunology

Received: 19 November 2020 Accepted: 03 February 2021

Published: 22 February 2021

Citation:

Kulkarni V, Queiroz ATL, Sangle S, Kagal A, Salvi S, Gupta A, Ellner J, Kadam D, Rolla VC, Andrade BB,

Salgame $P$ and Mave V (2021) A

Two-Gene Signature for Tuberculosis

Diagnosis in Persons With Advanced

HIV. Front. Immunol. 12:631165.

doi: 10.3389/fimmu.2021.631165
Background: Transcriptomic signatures for tuberculosis (TB) have been proposed and represent a promising diagnostic tool. Data remain limited in persons with advanced HIV.

Methods: We enrolled 30 patients with advanced HIV (CD4 < 100 cells $\left./ \mathrm{mm}^{3}\right)$ in India; 16 with active TB and 14 without. Whole-blood RNA sequencing was performed; these data were merged with a publicly available dataset from Uganda $(n=33 ; 18$ with TB and 15 without). Transcriptomic profiling and machine learning algorithms identified an optimal gene signature for TB classification. Receiver operating characteristic analysis was used to assess performance.

Results: Among 565 differentially expressed genes identified for TB, 40 were shared across India and Uganda cohorts. Common upregulated pathways reflect Toll-like receptor cascades and neutrophil degranulation. The machine-learning decision-tree algorithm selected gene expression values from RAB20 and INSL3 as most informative for TB classification. The signature accurately classified TB in discovery cohorts (India AUC 0.95 and Uganda AUC 1.0; $p<0.001$ ); accuracy was fair in external validation cohorts.

Conclusions: Expression values of RAB2O and INSL3 genes in peripheral blood compose a biosignature that accurately classified TB status among patients with advanced HIV in two geographically distinct cohorts. The functional analysis suggests pathways previously reported in TB pathogenesis.

Keywords: HIV, tuberculosis, transcriptomics, diagnosis, gene signature

\section{INTRODUCTION}

Tremendous advances in tuberculosis diagnosis have been made based on nucleic acid amplification of bacteria in the sputum, such as Xpert MTB/RIF sputum smear and culture, which provides results in $2 \mathrm{~h}(1-5)$. However, sputum-based diagnostics remain problematic in the context of HIV infection. Sputum smear is often negative for TB bacilli, and the sensitivity of Xpert 
MTB/RIF is only $67 \%$ (6-8). Persons living with advanced HIV $\left(\mathrm{CD} 4<100\right.$ cells $\left./ \mathrm{mm}^{3}\right)$ are at particularly high risk for TB and are likely to have smear-negative pulmonary or extrapulmonary $\mathrm{TB}$, underscoring the need for non-sputum-based TB diagnostics to support TB control efforts (9-15).

Blood-based transcriptomic signatures, including several parsimonious gene signatures, have been proposed to diagnose and differentiate $\mathrm{TB}$ from other respiratory diseases (ORD) and are in various stages of validation $(14,16,17)$. However, the majority of studies do not include persons living with advanced HIV. A recent case-control study from Uganda found that transcript levels of FcGR1A and BATF2 and plasma protein levels of interferon gamma (IFN- $\gamma$ ) and CXCL10 were individually accurate classifiers of active TB in the context of advanced HIV (18). However, geographic differences may exist and could impact performance when transcriptomic profiles developed in one population are applied to other geographically distinct populations.

To address the potential influence of geography and the reduced number of TB gene expression signatures addressing persons living with HIV (PLWH), we established a discovery cohort comprising the publicly available RNA sequencing (RNAseq) dataset from the aforementioned Uganda case-control study $(n=33)$ (18) and RNA-seq data from our prospective casecontrol study in India among persons with advanced HIV with or without active TB $(n=30)$. Using transcriptomic profiling and a machine-learning approach, we aimed to develop and validate a gene signature to fairly classify TB status among persons with advanced HIV from geographically distinct sites.

\section{METHODS}

\section{Discovery Cohorts} India Cohort

Between January 2018 and June 2019, we enrolled 30 consecutive adults attending the antiretroviral treatment (ART) clinic at Byramjee Jeejeebhoy Government Medical College (BJGMC) and Sassoon General Hospitals (SGH), which provides HIV care to residents of Pune, India, and the surrounding area. Eligibility criteria were ART-naïve and ART-experienced adults ( $>18$ years) with advanced HIV, defined as CD4 $<100$ cells $/ \mathrm{mm}^{3}$, with or without newly diagnosed active TB. Exclusion criteria were previous history of TB or anti-tuberculosis treatment (ATT) before enrolment. All potential participants underwent TB symptom screen and GeneXpert MTB/RIF, sputum smear and culture. Cases (TB-HIV), defined as any positive microbiologic TB investigations or ATT initiation based on high clinical suspicion (active TB), were enrolled up to $n=15$; controls (HIVonly), defined as no evidence of active TB, were enrolled up to $n$ $=15$. Medical, demographic, socio-economic characteristics, and chest radiograph were obtained at enrolment, and blood samples were collected at baseline for HIV quantitative RNA and CD4+

Abbreviations: ART, antiretroviral therapy; ATT, anti-tuberculosis treatment; AUC, area under the curve; DEG, differentially expressed genes; HIV, human immunodeficiency virus; TB, tuberculosis; PLWH, people living with HIV; PCA, principal component analysis.
T-cell count. Individual participant consent as well as BJGMC ethics committee and Johns Hopkins University institutional review committee approvals were obtained.

\section{Uganda Cohort}

A published case-control study conducted among 33 adults with advanced HIV (CD4 count $<100$ cells $/ \mathrm{mm}^{3}$ ) in Uganda. The study population comprised 18 cases with active TB (TBHIV; 16 with smear-positive or microbiologically-confirmed TB and 2 undergoing ATT) and 15 controls (HIV-only) with no clinical symptoms of TB. All participants underwent whole-blood RNA sequencing (RNA-seq) and plasma cytokine/chemokine analysis (18).

\section{Whole Blood Sample Processing and RNA Sequencing}

At enrolment, whole blood $(5 \mathrm{~mL})$ was collected from all 30 India participants in two PAXgene Blood RNA tubes (Qiagen, catalog $\# 762165$ ) and directly frozen at $-80^{\circ} \mathrm{C}$. RNA was extracted using the PAXgene Blood RNA kit (Qiagen, catalog \#762174) and quantified using Qubit RNA assay HS (Invitrogen, Cat \#Q32852). RNA purity was checked using QIAxpert, and RNA integrity was assessed on TapeStation using RNA HS ScreenTapes (Agilent, Cat \#5067-5579). NEB Ultra II Directional RNA-Seq Library Prep kit protocol was used to prepare libraries for total RNA sequencing. Prepared libraries were quantified using Qubit High Sensitivity Assay (Invitrogen, Cat \#Q32852), pooled and diluted to final optimal loading concentration before cluster amplification on Illumina flow cell. Once the cluster generation was completed, the cluster flow cell was loaded on Illumina HiSeqX instrument to generate $150 \mathrm{bp}$ paired-end reads.

\section{Gene Expression Analysis}

Raw RNA-seq data from the India cohort were retrieved from Illumina HiSeqX in fastq formatted files and processed using the protocol for paired-end reads in the quality check and mapping step; raw RNA-seq data from the Uganda cohort were downloaded from the NCBI SRA database using sra-tools (https://ncbi.github.io/sra-tools/fastq-dump.html) and processed using the single-end protocol in the quality check and mapping step. Low quality bases were removed from all samples, and adapters were trimmed using Trimmomatic V0.32 (19). A total of 5 samples failed in the quality check process from India Cohort and were removed from analysis. A total of 58 samples from both sites were used in downstream analysis. After the quality check, sequences were aligned to the human transcriptome (GRCh38 version 100), comprising mRNA and ncRNA, using Salmon v1.2.0 (20). After the mapping step, the Salmon output was converted to count tables using the tximport $\mathrm{R}$ package (21). Count gene expression matrix was examined using the DESeq2 R package (22) to identify differentially expressed genes (DEG) for cases. Changes in gene expression with false discovery rate (FDR)-adjusted $p$-value $<0.05$ and $\log _{2}$ fold-change \pm 1.4 were considered significant. Candidate DEGs were visualized using volcano plots and Venn diagrams using the VennDiagram $\mathrm{R}$ package and scanned with the REACTOME pathway database (23) using the compareCluster 
$\mathrm{R}$ package (24). The entire gene expression data set from India cohort is available at the GEO database (Accession number GSE162164, https://www.ncbi.nlm.nih.gov/geo/query/ acc.cgi? acc $=$ GSE162164).

\section{Machine Learning Approach}

Following variance-stabilizing transformation and batch effect correction [sva package (25)], gene expression measurements were used to perform a machine learning approach. Using the rpart R package (26), a decision-tree algorithm with leaveone-out cross-validation was applied to identify the minimal variable set (gene set) exhibiting higher classification power to describe cases. The resulting genes were retrieved from each dataset. Sample clustering and classification were assessed using Heatmaps and the Principal component analysis (PCA) plot and applied to the variance-stabilizing transformed gene expression values from each cohort.

\section{Signature Performance Analysis}

We conducted a performance comparison using 36 previously published gene expression signatures for TB diagnosis, progression and treatment provided by the TBSignatureProfiler package (https://github.com/compbiomed/TBSignatureProfiler). In addition, we have included Risk6 signature cohort for comparison (27) (Supplementary Table 1). We applied a general linear model to gene expression values from each signature gene. The outcomes were binarized to measure the sensitivity and specificity of classification, allowing us to measure each group rate and plot area under the curve (AUC) values to identify the best classifier.

\section{Validation of the Gene Signature}

To validate the gene signature, we applied the gene expression model to gene expression data, which was $\log 2$ normalized, from three independent and publicly available patient cohorts (28-30). The first study developed and validated transcriptomic signatures to distinguish TB from latent TB infection (LTBI) using a casecontrol design among African adults with and without HIV (28); validation was performed by comparing TB-HIV (with and without culture-confirmed TB) vs. HIV-only. The second study identified and validated transcriptomic signatures to distinguish active TB from other respiratory diseases as well as LTBI among large pediatric cohorts from South Africa, Malawi and Kenya (29); the comparison for validation was TB-HIV vs. HIV and other respiratory diseases.

\section{Statistical Analysis}

All analyses were pre-specified. Clinical data were compared among cases and controls using the Mann-Whitney $U$ test (continuous variables) or Pearson's chi-square test (categorical variables). Correlations between gene expression and clinical variables were tested using Spearman's rank correlation coefficient. Receiver Operator Characteristics (ROC) were used to assess the accuracy of a gene signature to distinguish between comparison groups specified in the India/Uganda datasets and each validation dataset (in-silico validation cohorts). We measured the $\mathrm{z}$-scores with the scales function. Analyses were
TABLE 1 | Baseline characteristics among cases (TB-HIV) and controls (HIV-only) enrolled in the India cohort $(n=30)$.

\begin{tabular}{|c|c|c|c|}
\hline Characteristic & $\begin{array}{l}\text { HIV-only } \\
(n=14)\end{array}$ & $\begin{array}{c}\text { TB-HIV } \\
(n=16)\end{array}$ & $p$-value \\
\hline \multicolumn{4}{|l|}{ Sex } \\
\hline Female, n (\%) & $4(29 \%)$ & $3(18 \%)$ & 0.68 \\
\hline Male, n (\%) & $10(71 \%)$ & $13(82 \%)$ & \\
\hline Median age, y (IQR) & $41(31-52)$ & 45 (38-52) & 0.42 \\
\hline \multicolumn{4}{|l|}{ Smoker, n (\%) } \\
\hline Never & $10(71 \%)$ & $11(69 \%)$ & $>0.95$ \\
\hline Former & 0 & $1(6 \%)$ & \\
\hline Current & $4(29 \%)$ & $4(25 \%)$ & \\
\hline Body mass index, $\mathrm{kg} / \mathrm{m}^{2}$ & $\begin{array}{c}20.0 \\
(16.8-21.4)\end{array}$ & $\begin{array}{c}17.6 \\
(16.4-19.9)\end{array}$ & 0.23 \\
\hline Median HIV viral load, $\log _{10}$ copies $/ \mathrm{mL}$ & $\begin{array}{c}4.92 \\
(4.24-5.77)\end{array}$ & $\begin{array}{c}5.50 \\
(4.97-5.87)\end{array}$ & 0.32 \\
\hline Median CD4 count, cells/mm³ (IQR) & $53(32-75)$ & $48(31-65)$ & 0.60 \\
\hline Median CD8 count, cells/mm³ (IQR) & $\begin{array}{c}645 \\
(320-861)\end{array}$ & $\begin{array}{c}430 \\
(244-589)\end{array}$ & 0.13 \\
\hline Median CD3 count, cells/mm³ (IQR) & $\begin{array}{c}739.5 \\
(407-1,043)\end{array}$ & $\begin{array}{c}491 \\
(290-679.5)\end{array}$ & 0.15 \\
\hline
\end{tabular}

BMI, body mass index; HIV, human immunodeficiency virus; TB, tuberculosis.

performed using the base package from $\mathrm{R}$ 4.0.2. Differences with $p$-values $<0.05$ were considered statistically significant.

\section{RESULTS}

\section{Description of Discovery Cohorts}

Cases $(n=16)$ and controls $(n=14)$ from the India cohort $(n$ $=30$ ) did not significantly differ among baseline characteristics, including sex ( $82 \%$ male vs. $71 \%$ male), median age (45 vs. 41 years), median CD4 count ( 45 vs. 53 cells $/ \mathrm{mm}^{3}$ ) and median HIV viral load (5.50 vs. $4.92 \log$ copies $/ \mathrm{mL}$ ) (Table 1). The Uganda cohort $(n=33)$ was $62 \%$ female, median age was 32 years and median CD4 count was 50 cells $/ \mathrm{mm}^{3}$ with no significant differences between cases $(n=18)$ and controls $(n=15)(18)$.

\section{Gene Expression Analysis}

A total of 565 DEGs were identified for cases (active TB) among the discovery cohorts. Of these, the majority (488 DEGs) were specific to the Uganda cohort, including 265 upregulated and 223 downregulated genes; 37 were specific to the India cohort, including 32 upregulated and 5 downregulated genes; and 40 were shared by both cohorts (Supplementary Figure 1, Supplementary Table 2). Cluster analysis revealed that DEGs identified at each site were able to distinguish samples from cases and controls, but with some misclassifications (Supplementary Figure 2).

The majority of shared DEGs were upregulated (38 upregulated vs. 2 downregulated). The enrichment analysis shown in Figure 1 reveals that only two pathways were enriched in both discovery cohorts, namely Toll-like receptor cascades and Neutrophil degranulation. Among Ugandaspecific DEGs, upregulated pathways predominantly reflect 


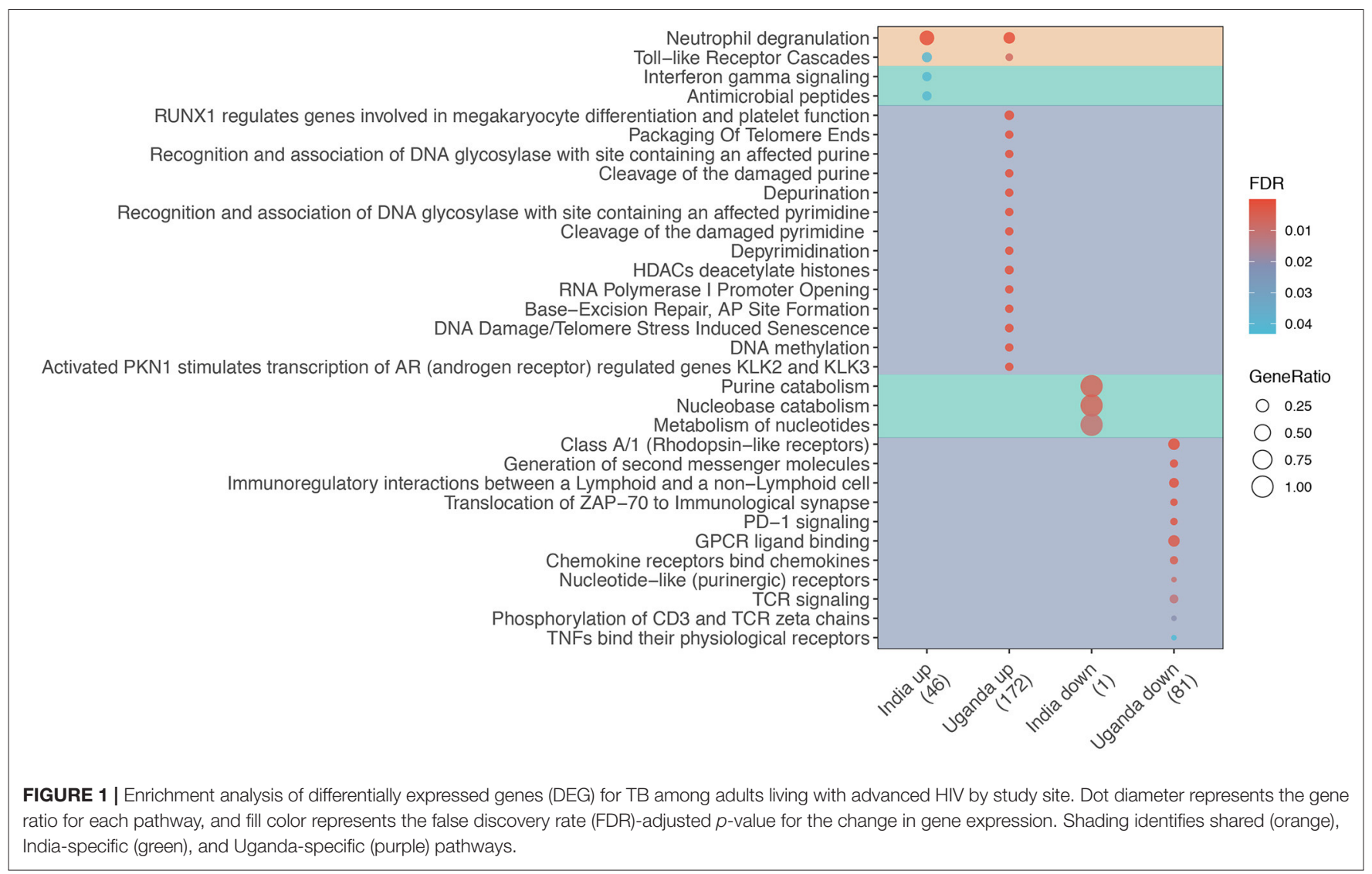

DNA repair and regulation, and downregulated pathways reflect immune cell response regulation. In contrast, Indiaspecific upregulated pathways reflect IFN- $\gamma$ signaling and antimicrobial peptide response while downregulated pathways reflect nucleotide metabolism.

\section{Machine Learning}

Gene expression values from DEGs were used to perform machine learning. The decision tree identified INSL3 and RAB20 (Decision-tree genes) as the optimal gene set to classify tuberculosis status among patients from both sites (Figure 2A). Dot plots show that threshold gene expression values for INSL3 and $R A B 20$ fairly classified samples from both study sites, correctly classifying $100 \%$ of Uganda samples and returning only 3 classification errors in the India cohort (Figures 2B,C). Receiver operator characteristic (ROC) analysis indicates accurate TB classification among samples from India [AUC 0.95 (0.87-1.00)] and Uganda (AUC 1.00) (Figure 2D). Compared to DEGs and 36 proposed TB gene expression signatures, the Decision-tree genes best classified TB status among samples from both cohorts (Figures 2E,F). Although the Maertzdorf_4, Roe_3 and Suliman_4 signatures and Decision-tree genes performed comparably in the India cohort, the Maertzdorf and Suliman signatures comprises 4 genes and Roe signature comprises 3 genes, and was not as accurate in the Uganda cohort where the Rajan_HIV_5 and Decision-tree signatures performed best. Reviewing potential associations between Decision-tree genes and previously proposed $\mathrm{TB}$ signatures revealed that $R A B 20$ is included in the Bankley_380 (383 genes) and Barry_393 (290 genes) signatures (Supplementary Figure 3A), yet the Decision-tree genes had superior performance in both cohorts.

\section{Correlation of Clinical Variables With Decision-Tree Gene Expression}

Among the India cohort, CD8+ and CD3+ cell counts were significantly lower in cases than controls (Supplementary Figures 3B-D). Comparing Decision-tree gene expression to clinical variables, Spearman correlation values indicate a significant negative correlation between INSL3 expression and both CD8+ and CD3+ cell counts. No cluster was associated with clinical variables (Smoke, Cough, Cavitation, Death, Viral load, CD4, Age or BMI) (Figure 3).

\section{Validation of the Decision-Tree Signature}

We performed ROC analysis to determine the sensitivity of the 2-gene signature to distinguish active $\mathrm{TB}$ among three validation cohorts. As shown in Figure 4, the Decision-tree signature performed best among South African cohorts with AUC ranging between 0.683 and 0.748 ; performance was lower among Malawi cohorts with AUC ranging between 0.615 and 0.623 (Figures 4A,B). The 2-gene signature demonstrated high accuracy to predict active TB with an AUC of 0.945 for distinguishing culture-confirmed TB from culture-negative TB (Figure 4C). 


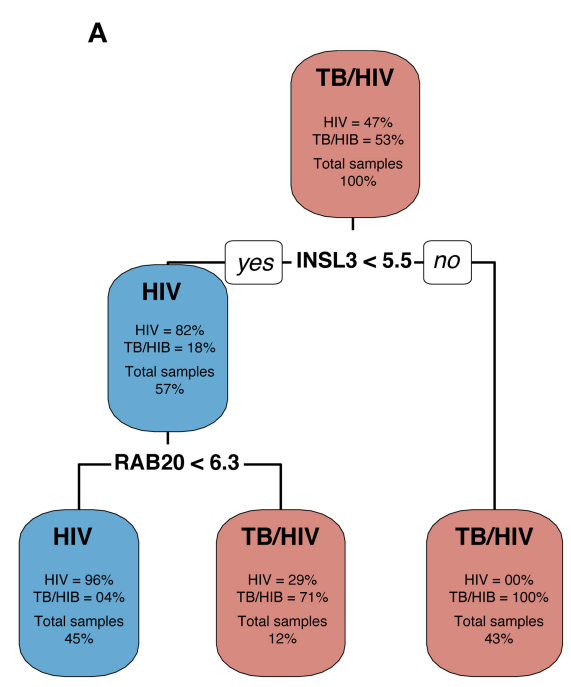

E
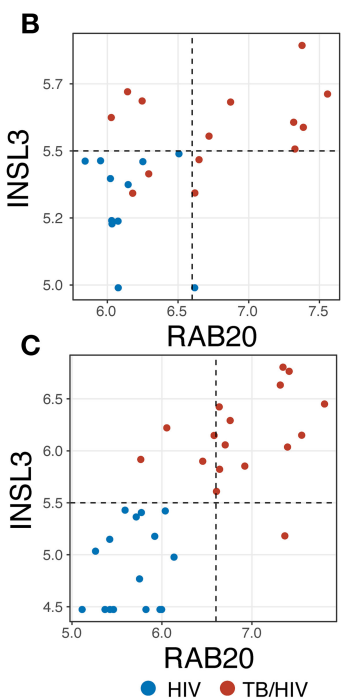

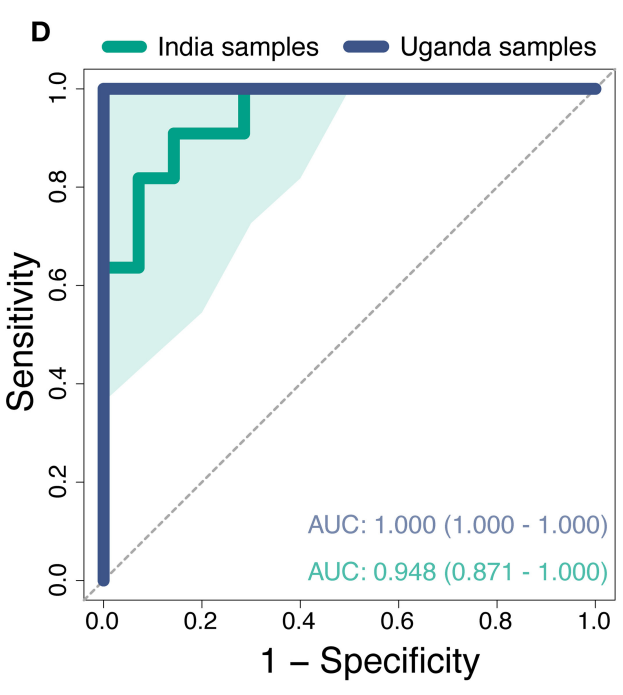

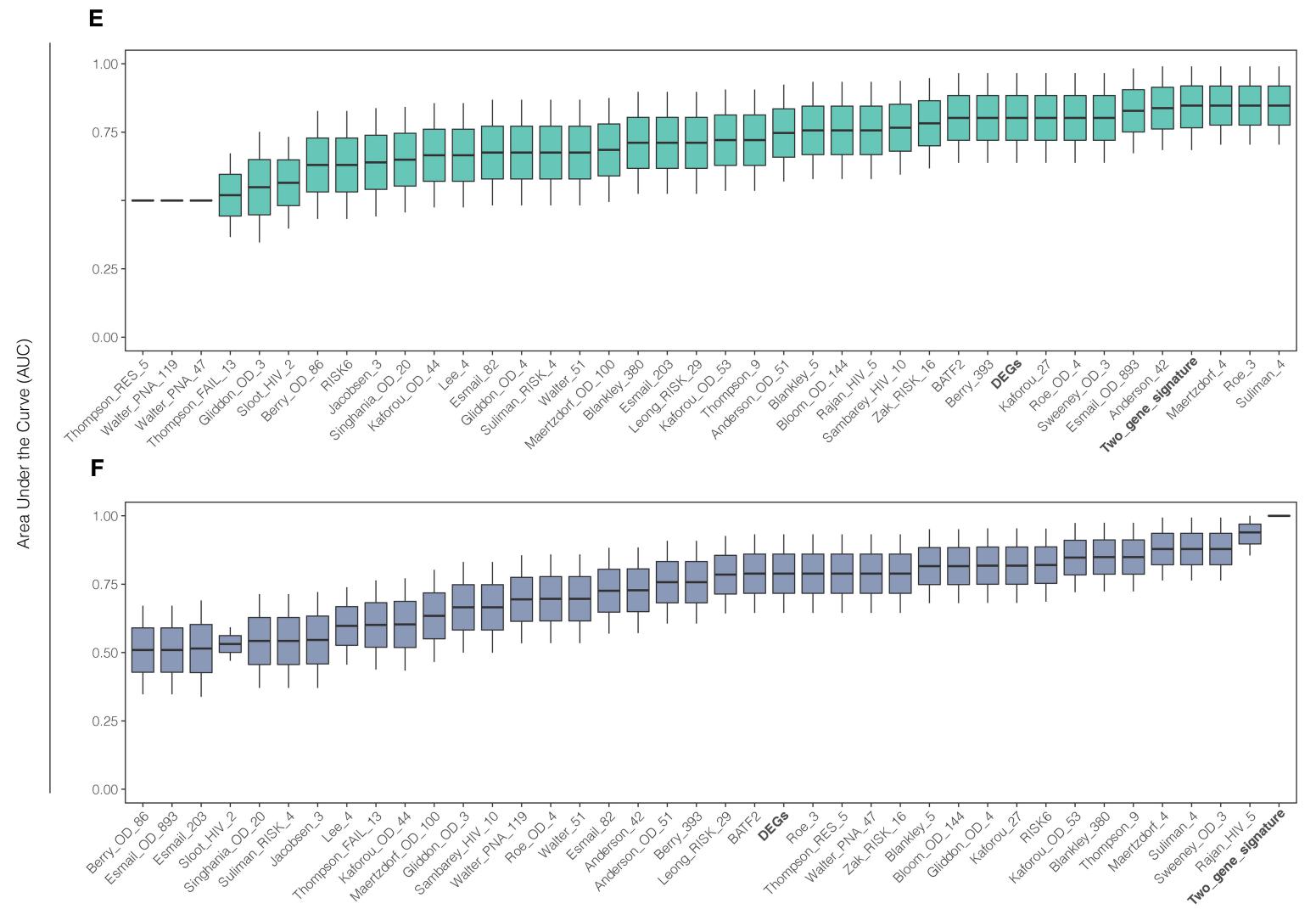

FIGURE 2 | The machine learning approach identified a 2-gene signature (INSL3 and RAB20) that best classified tuberculosis status across study sites. (A) The decision-tree algorithm selected INSL3 and RAB20 genes to classify tuberculosis status among the discovery cohorts. (B,C) Dot plots show that Decision-tree genes correctly classify TB status for most samples from the India cohort (B) and for 100\% of samples from the Uganda cohort (C); vertical and horizontal dotted lines represent decision thresholds for RAB20 and INSL3 genes, respectively. (D) Receiver operating characteristic (ROC) curve analysis shows strong TB classification performance of Decision-tree genes among samples from India (green line) and Uganda (purple line) with area under the curve (AUC) of 0.948 and 1.00, respectively; shaded area represents standard deviation. Boxplots show the AUC, measured by general linear modeling, for Decision-tree genes (Bold), differentially expressed genes (Bold), and publicly available TB gene expression signatures identifying the Decision-tree genes as the best TB classifier across India (E) and Uganda (F) cohorts. 


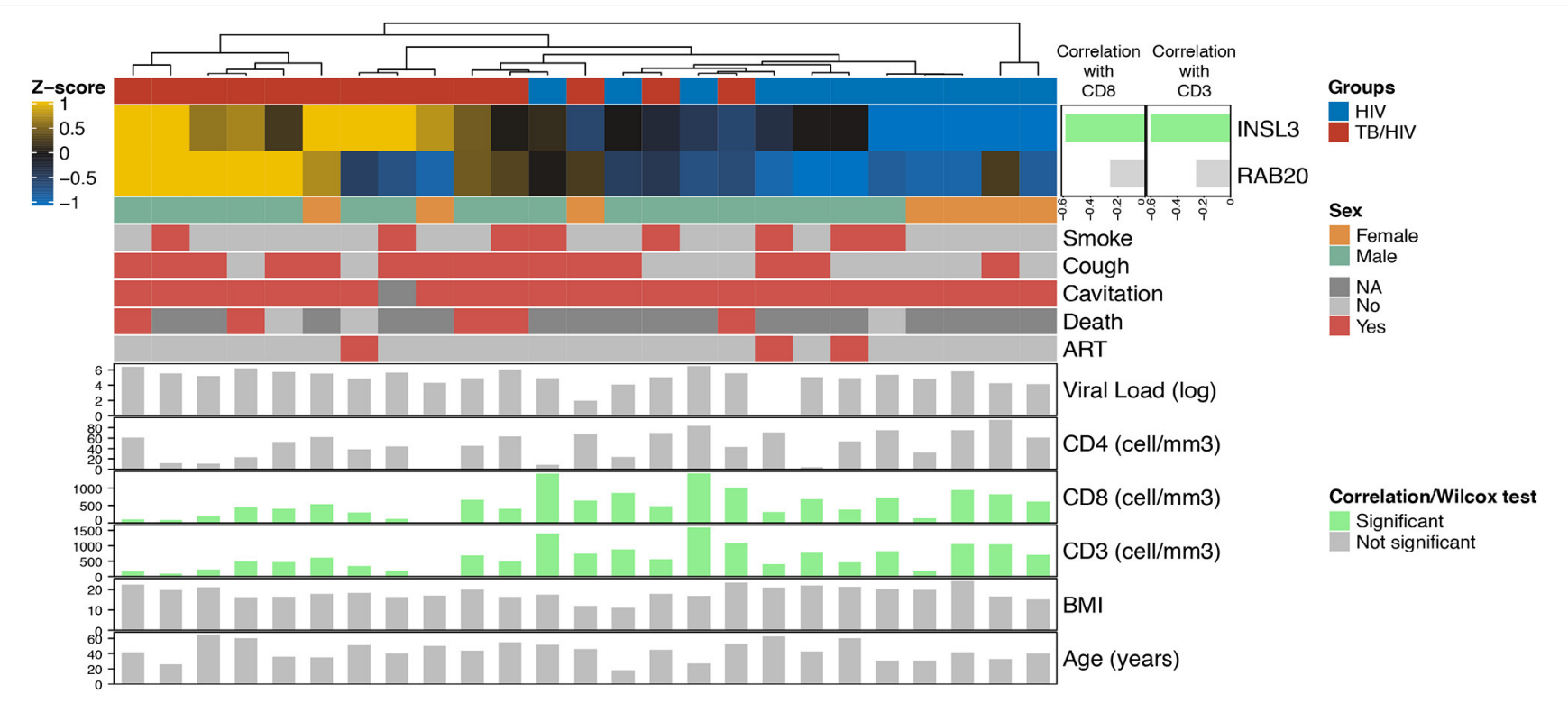

FIGURE 3 | Heatmap showing the relationship between Decision-tree gene expression and clinical characteristics in the India cohort. The top horizontal bar corresponds to cases (red) and controls (blue). The side bar plot shows the Spearman correlation value measuring the association between INLS3 and RAB20 expression and CD8+ and CD3+ cell count; green bars indicate a significant association. The lower horizontal bars correspond to female (orange) vs. male (green) followed presence (red) vs. absence (light gray) of participant characteristics; dark gray indicates no information available. The bottom bar plots show significant (green vertical bars) and non-significant (gray vertical bars) correlations with participant characteristics, including log 2 HIV viral load; CD4+/CD8+/CD3+ cell counts, body mass index (BMI) and age.

\section{DISCUSSION}

Transcriptomic signatures for TB diagnosis have been previously identified using various approaches, including differentially expressed genes, pathway analysis and subsetting genes associated to symptomatology $(15,16,31)$. Although the blood transcriptomic profiling can improve diagnosis and understanding of $\mathrm{TB}$ infection, population-specific gene expression could interfere with performance across different regions (32). This study identified a 2-gene parsimonious signature that accurately classified active TB among people with advanced HIV infection in two geographically distinct cohorts. More importantly, the signature fared well to distinguish active TB from latent tuberculosis infection (LTBI) as well as other respiratory diseases when applied to other African datasets. Finally, the signature performed best among those with culture-confirmed TB and is likely an indicator of mycobacterial replication, suggesting the potential to extrapolate its use for TB treatment monitoring.

The prediction of TB diagnosis in PLWH improved when Indian and Ugandan datasets were combined. The two genes generated by the machine learning algorithm (RAB20 and INSL3) were able to accurately distinguish active TB from non-TB. $R A B 20$, a member of the RAS Oncogene Family, is involved in the maturation and acidification of phagosomes. More specifically, $R A B 20$ regulates the endosomal membrane, thus playing an important role in phagosome integrity and control of Mycobacterium tuberculosis (Mtb) replication in infected macrophages (33). This mechanism is also regulated by IFN$\gamma$, assisting with $M t b$ infection control in macrophages (34). In contrast, INSL3 is part of an insulin-like hormone superfamily and is associated with human testicular cell tumors (35), but has not been previously associated with TB infection or disease. Notably, the strong negative correlation observed between INSL3 expression and CD8+/CD3+ cell count (rho-0.6) suggests a significant role in immune cell regulation among PLWH with active TB from India. The influence of INSL3 on CD8+ and CD3 + cells could be associated with its regulation of TIMP2 (36), a member of the NF-KappaB Family Pathway.

Although the 2-gene signature performed well in both discovery cohorts, we observed considerable geographic differences in gene expression between India and Uganda. Specifically, samples from Africa presented more DEGs (528 genes) than India (77 genes), and only 40 common DEGs were identified across the sites. A multitude of factors alter the immune response and may explain the observed differences, including ethnic population, dietary, environmental and seasonal differences $(37,38)$. Variable performance of TB signatures in Indian and African discovery cohorts provides additional evidence of population-specific gene expression. The performance of TB signatures varied with lower AUC observed among India samples compared to Uganda. Even signatures proposed among PLWH, such as Esmail_82, Esmail_203, Esmail_893 (31), Kaforou_27, Kaforou_OD_44, Kaforou_OD_53 (28), Sambarey_HIV_10 (39), and Rajan_HIV_5 (40), demonstrated differential performance 
among Indian and Ugandan cohorts. The total number of genes varies widely across signatures, ranging from 5 to 893 genes, and could explain the differential performance (AUC) in classifying TB status among PLWH, but also suggests a possible population bias in each signature that could interfere with its use in other geographic locations.

The differential gene expressions observed between Indian and Ugandan cohorts was not unexpected. Despite the differences, however, the discovery cohorts shared 40 differentially expressed genes for $\mathrm{TB}$, and two important pathways were found to be upregulated in both cohorts (18). The Toll-like receptor cascade pathway has been previously associated with TB and HIV, indicating the role of $M t b$ in the regulation of HIV replication (41). The neutrophil degranulation pathway has also been associated with $\mathrm{TB}$, but the exact role of neutrophils remains ambiguous with potential to be associated with $M t b$ clearance as well as increased disease severity and mortality (42). Overall, these pathways suggest that TB disease may influence peripheral blood mononuclear cell expression in PLWH.

The performance of the novel 2-gene signature is heterogeneous in the external validation data sets, but the 2-gene signature has fair overall accuracy to distinguish TB. Accuracy ranged from 0.683 to 0.748 in the African cohort comprising children and adults, and inferior performance was observed in the Malawi cohort with AUC values ranging from 0.615 to 0.623 . The difference in performance suggests that population-associated gene expression interferes with TB classification in PLWH. Despite the unsatisfactory performance of the 2-gene signature in these data, some aspects should be accounted. In this dataset, the control group was composed of PLWH and other respiratory diseases. The control group composition and population bias may have contributed to reduced AUC values. Interestingly, TB classification accuracy was high for patients with culture-confirmed TB in the Kenya cohort (AUC 0.954) while reduced performance was observed among patients without culture-confirmed TB (AUC 0.627). This finding suggests an association of the two-gene signature with bacterial load and that longitudinal change in expression of this gene signature could also be used to monitor bacillary load in response to treatment.

Gene signatures derived from multiple cohorts were validated using a targeted approach, reverse transcriptase multiplex ligation-dependent probe amplification (RT-MLPA) in a multisite study that comprised cohorts with and without HIV. The analysis revealed FCGR1A [high-affinity IgG Fc receptor 1 (CD64)] as a consistent single-gene classifier of active TB disease, in the presence and absence of HIV (43). FcGR1A was also reported to function as a consistent single gene classifier of active TB even in advanced HIV in the Uganda cohort included in this study (18). In an Ethiopian cohort, five genes (CD8A, TIMP2, CCL22, FCGR1A, and TNFRSF1A), were shown to segregate active $\mathrm{TB}$ from non-active $\mathrm{TB}$ in HIV patients (44). In another study, also in an Ethiopian cohort of HIV co-infected TB patients, 7 genes (FCGR1A, RAB24, TLR1, TLR4, MMP9, NLRC4, and IL1B) accurately discriminated between active tuberculosis disease and latent

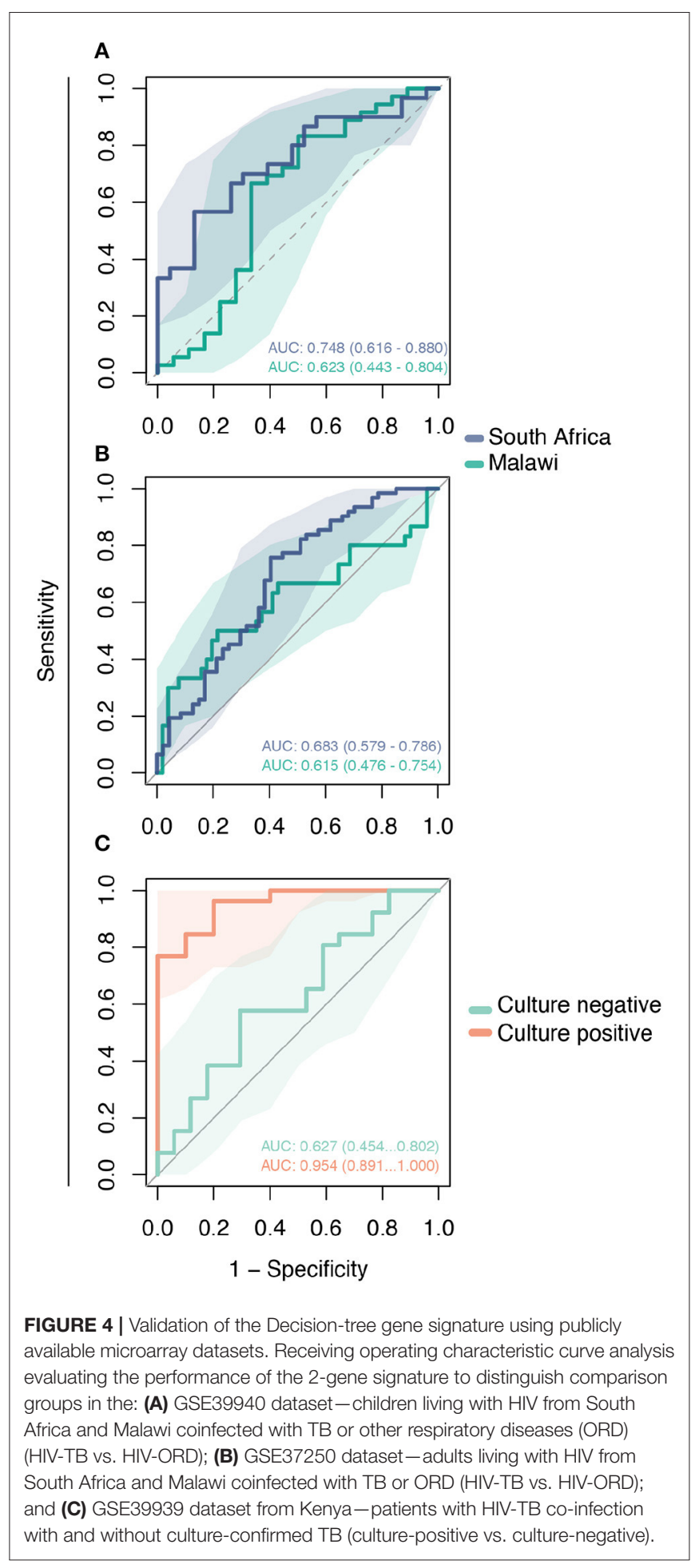

infection (45). RISK6 is a prognostic signature derived from baseline blood samples in a SA adolescent cohort of progressors and non-progressors (27). The signature is an aggregate of nine transcript pairs that was derived by separately linking each of three transcripts upregulated in progressors (GBP2, 
FCGR1B, and SERPING1), to three transcripts downregulated in progressors (TUBGCP6, TRMT2A, and SDR39U1), relative to non-progressors. RISK6 also performed well in diagnosing active TB in HIV-uninfected and HIV-infected persons (27). Of note, none of the studies included cohorts from India. Additional head-to-head comparative studies in larger cohorts are needed to determine whether the 2-gene signature reported here works across ethnicities and comorbidities, including HIV. Furthermore, whether the same gene signatures will perform well in segregating TB from HIV with differing CD4 counts and differing peripheral inflammation also needs to be determined.

Despite yielding interesting results, our study has some limitations. First, the sampling size is not ideal, with 25 samples from India and 33 from Uganda, and has resulted in more variability observed in the study. Second, the metadata from all validation datasets do not have the CD4 count value for each patient, but the overall cohort data report much higher CD4 value than our cohort. This may have contributed to reduction in performance of our signature. For clinical application, more studies are required to standardize a gene expression-based protocol. Furthermore, RNA seq-based signatures need to be further developed for use in clinical practice to distinguish PLWH with TB from those with LTBI or other respiratory diseases.

In conclusion, despite populational-specific differential gene expression, the RAB20 and INSL3 genes outperformed all previously proposed $\mathrm{TB}$ signatures to accurately distinguish TB from non-TB among multiple cohorts from different geographical regions. This parsimonious 2-gene signature also performed well among those with culture-positive TB, indicating its potential use for TB treatment monitoring. Our study provides evidence supporting a promising, novel and non-sputum-based biomarker for TB diagnosis, especially for those with advanced HIV infection in whom TB diagnosis is often difficult with sputum-based diagnostics. Future studies are needed to confirm our findings.

\section{DATA AVAILABILITY STATEMENT}

The datasets presented in this study can be found in online repositories. The names of the repository and accession number can be found here: GEO database (Accession number GSE162164, https://www.ncbi.nlm.nih.gov/geo/query/acc.cgi? acc $=$ GSE162164).

\section{ETHICS STATEMENT}

The studies involving human participants were reviewed and approved by (1) Byramjee Jeejeebhoy Government Medical College Clinical Trials Unit. (2) INI-FIOCRUZ, Brazil; FMT, Brazil. (3) Johns Hopkins University School of Medicine, USA. (4) Boston Medical Campus (BUMC), USA. (5) Rutgers New Jersey Medical School IRB, USA. The patients/participants provided their written informed consent to participate in this study.

\section{AUTHOR CONTRIBUTIONS}

VM, DK, VR, PS, and JE contributed to study design. VK, AQ, and BA contributed toward data acquisition. PS, AQ, and BA contributed equally toward data interpretation. VK, SSan, AK, SSal, DK, and VM were responsible for patient recruitment, sample collection, storage, and analysis of clinical data. VK, AQ, VM, PS, and BA contributed equally to writing the manuscript. All authors read and approved the final manuscript.

\section{FUNDING}

The research work was primarily supported by CRDF Global (Award number DAA3-17-63158-1/Country: India). Research data in this manuscript were collected, in part, as part of the Regional Prospective Observational Research for Tuberculosis (RePORT) India Consortium. This work was funded in whole or in part by federal funds from the Government of India Department of Biotechnology (DBT); the Indian Council of Medical Research (ICMR); the National Institutes of Health National Institute of Allergy and Infectious Diseases Office of AIDS Research; CRDF Global; the National Institutes of Health Baltimore-Washington-India Clinical Trials Unit for National Institute of Allergy and Infectious Diseases Networks (UM1AI069465 to VM and AG); and the National Institutes of Health (R01A1I097494 to VM and AG). The work of BBA was supported by the Intramural Research Program of the Oswaldo Cruz Foundation, and the National Council for Scientific and Technological Development (senior CNPq fellowship-tier 1D), Brazil. The contents of this publication are solely the responsibility of the authors and do not represent the official views of the DBT, ICMR, National Institutes of Health, or CRDF Global. Any mention of trade names, commercial projects, or organizations does not imply endorsement by any of the sponsoring organizations. The content of this paper is solely the responsibility of the authors and does not represent the official views of the funders.

\section{ACKNOWLEDGMENTS}

We would like to thank the study participants, ART center nurses, study counselor, and laboratory staff for their efforts in enrollment, sample processing, storage and shipment.

\section{SUPPLEMENTARY MATERIAL}

The Supplementary Material for this article can be found online at: https://www.frontiersin.org/articles/10.3389/fimmu. 2021.631165/full\#supplementary-material

Supplementary Figure S1 | (A,B) Volcano plots showing differentially expressed genes (DEG) for TB using whole-blood samples from India (A) and Uganda (B) cohorts. Red indicates DEGs, defined as change in gene expression with $\log _{2}$ Fold Change \pm 1.4 and FDR $<0.05$; green indicates change in gene expression with $\log _{2}$ Fold Change \pm 1.4 ; blue indicates change in gene expression with FDR $<0.05$; and gray indicates no significant change in gene expression. (C) The Venn diagram shows the number of site-specific and shared DEGS. (D) The Bar plot shows the $\log _{2}$ Fold Change of the 40 DEGs shared across study sites. 
Supplementary Figure $\mathbf{S 2}$ | Heatmap of the differentially expressed genes (DEG) for TB identified in the India (A) and Uganda (B) cohorts.

Supplementary Figure S3 | The dot plot demonstrates the presence of Decision-tree genes (INSL3 and RAB20) in previously proposed TB gene expression signatures (A). Boxplots show the associations of CD3 (B), CD8 (B), and CD4 (C) cell counts and HIV viral load (D) with TB-HIV co-infection status in the India cohort. Clinical variables were compared among cases (TB-HIV) and controls (HIV-only) using the Wilcoxon test. Only CD3 and CD8 cell counts were significantly associated with TB status.
Supplementary Table 1 | Systematic literature review but restricted to the signatures present in TBSiginatureProfiler package, plus the RISK6 used as reference. Signature names represent the first author's name of the corresponding publication, suffixed with number of constituent genes that are present in the RNAseq dataset. Table includes number of genes, method, population, HIV status and treatment, geographical region, and participant condition. TB, tuberculosis; LTBI, latent tuberculosis infection; HHC, household contacts; SARC, sarcoidosis.

Supplementary Table 2 | File with the differentially expressed genes (DEG) list from India, Uganda cohort, and the common genes. The log2 Fold Change, log2 Fold Change SE, p-value, and FDR are also provided for each gene.

\section{REFERENCES}

1. Kebede A, Beyene D, Yenew B, Diriba G, Mehamd Z, Alemu A, et al. Monitoring quality indicators for the Xpert MTB/RIF molecular assay in Ethiopia. PLoS ONE. (2019) 14:e0225205. doi: 10.1371/journal.pone.0225205

2. Lawn SD, Nicol MP. Xpert $\AA$ MTB/RIF assay: development, evaluation and implementation of a new rapid molecular diagnostic for tuberculosis and rifampicin resistance. Future Microbiol. (2011) 6:1067-82. doi: 10.2217/fmb.11.84

3. Vittor AY, Garland JM, Gilman RH. Molecular diagnosis of TB in the HIV positive population. Ann. Glob. Health. (2015) 80:476. doi: 10.1016/j.aogh.2015.01.001

4. Yusuf NW. Rapid diagnosis of tuberculosis using Xpert MTB/RIF assay-report from a third world country. Pak. J. Med. Sci. 31:10510. doi: $10.12669 /$ pjms. 311.6970

5. Agizew T, Chihota V, Nyirenda S, Tedla Z, Auld AF, Mathebula U, et al. Tuberculosis treatment outcomes among people living with HIV diagnosed using Xpert MTB/RIF versus sputum-smear microscopy in Botswana: a stepped-wedge cluster randomised trial. BMC Infect. Dis. (2019) 19:1058. doi: 10.1186/s12879-019-4697-5

6. Akanbi MO, Achenbach $\mathrm{C}$, Taiwo B, Idoko J, Ani A, Isa $\mathrm{Y}$, et al. Evaluation of gene Xpert for routine diagnosis of HIV-associated tuberculosis in Nigeria: aprospective cohort study. BMC Pulm. Med. (2017) 17:87. doi: 10.1186/s12890-017-0430-6

7. Sedky M, Wakil IA, Rashed M, Salama A. The role of genexpert in diagnosis of sputum-negative pulmonary tuberculosis. Egypt. J. Chest Dis. Tuberc. (2018) 67:419-26. doi: 10.4103/ejcdt.ejcdt_57_18

8. Steingart KR, Schiller I, Horne DJ, Pai M, Boehme CC, Dendukuri, N. Xpert ${ }^{\circledR}$ MTB/RIF assay for pulmonary tuberculosis and rifampicin resistance in adults. Cochrane Database Syst. Rev. (2014) 2014:CD009593. doi: 10.1002/14651858.CD009593.pub3

9. Blankley S, Graham CM, Turner J, Berry MPR, Bloom CI, et al. The transcriptional signature of active tuberculosis reflects symptom status in extra-pulmonary and pulmonary tuberculosis. PLoS ONE. (2016) 11:e0162220. doi: 10.1371/journal.pone.0162220

10. Burel JG, Babor M, Pomaznoy M, Lindestam Arlehamn CS, Khan N, Sette A. Host transcriptomics as a tool to identify diagnostic and mechanistic immune signatures of tuberculosis. Front. Immunol. (2019) 10:221. doi: 10.3389/fimmu.2019.00221

11. Darboe F, Mbandi SK, Naidoo K, Yende-Zuma N, Lewis L, Thompson EG, et al. Detection of tuberculosis recurrence, diagnosis and treatment response by a blood transcriptomic risk signature in HIVinfected persons on antiretroviral therapy. Front. Microbiol. (2019) 10:1441. doi: 10.3389/fmicb.2019.01441

12. Mulenga H, Bunyasi EW, Mbandi SK, Mendelsohn SC, Kagina B, PennNicholson A, et al. Performance of host blood transcriptomic signatures for diagnosing and predicting progression to tuberculosis disease in HIVnegative adults and adolescents: a systematic review protocol. BMJ Open. (2019) 9:e026612. doi: 10.1136/bmjopen-2018-026612

13. Turner CT, Gupta RK, Tsaliki E, Roe JK, Mondal P, Nyawo GR, et al. Blood transcriptional biomarkers for active pulmonary tuberculosis in a high-burden setting: a prospective, observational, diagnostic accuracy study. Lancet Respir. Med. (2020) 8:407-19. doi: 10.1016/S2213-2600(19)3 0469-2

14. Zak DE, Penn-Nicholson A, Scriba TJ, Thompson E, Suliman S, Amon LM, et al. A blood RNA signature for tuberculosis disease risk: a prospective cohort study. Lancet. (2016) 387:2312-22. doi: 10.1016/S0140-6736(15)01316-1

15. Sweeney TE, Braviak L, Tato CM, Khatri, P. Genome-wide expression for diagnosis of pulmonary tuberculosis: a multicohort analysis. Lancet Respir. Med. (2016) 4:213-24. doi: 10.1016/S2213-2600(16)00048-5

16. Singhania A, Wilkinson RJ, Rodrigue M, Haldar P, O'Garra, A. The value of transcriptomics in advancing knowledge of the immune response and diagnosis in tuberculosis. Nat. Immunol. (2018) 19:1159-68. doi: 10.1038/s41590-018-0225-9

17. Warsinske H, Vashisht R, Khatri, P. Host-response-based gene signatures for tuberculosis diagnosis: a systematic comparison of 16 signatures. PLoS Med. (2019) 16:e1002786. doi: 10.1371/journal.pmed.1002786

18. Verma S, Du P, Nakanjako D, Hermans S, Briggs J, Nakiyingi L, et al. Tuberculosis in advanced HIV infection is associated with increased expression of IFN $\gamma$ and its downstream targets. BMC Infect. Dis. (2018) 18:220. doi: 10.1186/s12879-018-3127-4

19. Bolger AM, Lohse M, Usadel B. Trimmomatic: a flexible trimmer for illumina sequence data. Bioinformatics. (2014) 30:2114-20. doi: 10.1093/bioinformatics/btu170

20. Patro R, Duggel G, Love MI, Irizarry RA, Kingsford C. Salmon provides fast and bias-aware quantification of transcript expression. Nat. Methods. (2017) 14:417-9. doi: 10.1038/nmeth.4197

21. Soneson C, Love MI, Robinson MD. Differential analyses for RNA-seq: transcript-level estimates improve gene-level inferences. F1000Res. (2015) 4:1521. doi: 10.12688/f1000research.7563.1

22. Love MI, Huber W, Anders S. Moderated estimation of fold change and dispersion for RNA-seq data with DESeq2. Genome Biol. (2014) 15:550. doi: 10.1186/s13059-014-0550-8

23. $\mathrm{Yu} \mathrm{G}, \mathrm{He} \mathrm{Q}-\mathrm{Y}$. ReactomePA: an R/Bioconductor package for reactome pathway analysis and visualization. Mol. Biosyst. (2016) 12:477-9. doi: 10.1039/C5MB00663E

24. Yu G, Wang L-G, Han Y, He Q-Y. clusterProfiler: an R package for comparing biological themes among gene clusters. OMICS. (2012) 16:2847. doi: $10.1089 /$ omi.2011.0118

25. Leek JT, Johnson WE, Parker HS, Fertig EJ, Jaffe AE, Zhang Y, et al. sva: Surrogate Variable Analysis. R package version 3.38.0 (2020).

26. Therneau T, Atkinson B, Ripley B. rpart: Recursive Partitioning and Regression Trees. R package version 4.1-102015 (2019).

27. Penn-Nicholson A, Mbandi SK, Thompson E, Mendelsohn SC, Suliman $\mathrm{S}$, Chegou NN, et al. RISK6, a 6-gene transcriptomic signature of TB disease risk, diagnosis and treatment response. Sci. Rep. (2020) 10:8629. doi: 10.1038/s41598-020-65043-8

28. Kaforou M, Wright VJ, Oni T, French N, Anderson ST, Bangani N, et al. Detection of tuberculosis in HIV-infected and -uninfected african adults using whole blood RNA expression signatures: a case-control study. PLoS Med. (2013) 10:e1001538. doi: 10.1371/journal.pmed.1001538

29. Anderson ST, Kaforou M, Brent AJ, Wright VJ, Banwell CM, Chagaluka G, et al. Diagnosis of childhood tuberculosis and host RNA expression in Africa. N. Engl. J. Med. (2014) 370:1712-23. doi: 10.1056/NEJMoa1303657

30. Marais S Lai RPJ, Wilkinson KA, Meintjes G, O'Garra A, Wilkinson RJ. Inflammasome activation underlying central nervous system deterioration in HIV-associated tuberculosis. J. Infect. Dis. (2017) 215:677-86. doi: 10.1093/infdis/jiw561 
31. Esmail H, Lai RP, Lesosky M, Wilkinson KA, Graham CM, Horswell S, et al. Complement pathway gene activation and rising circulating immune complexes characterize early disease in HIV-associated tuberculosis. Proc. Natl. Acad. Sci. U.S.A. (2018) 115:E964. doi: 10.1073/pnas.1711853115

32. Wang L, Rishishwar L, Mariño-Ramírez L, Jordan IK. Human population-specific gene expression and transcriptional network modification with polymorphic transposable elements. Nucl. Acids Res. 45:2318-28. doi: 10.1093/nar/gkw1286

33. Schnettger L, Rodgers A, Repnik U, Lai RP, Pei G, Verdoes M, et al. A Rab20dependent membrane trafficking pathway controls m. tuberculosis replication by regulating phagosome spaciousness and integrity. Cell Host Microbe. (2016) 21:619-28.e5. doi: 10.1016/j.chom.2017.04.004

34. Das R, Koo M-S, Kim BH, Jacob ST, Subbian S, Yao J, et al. Macrophage migration inhibitory factor (MIF) is a critical mediator of the innate immune response to Mycobacterium tuberculosis. Proc. Natl. Acad. Sci. U.S.A. (2013) 110:E2997-3006. doi: 10.1073/pnas.1301128110

35. Rossato M, Tavolini IM, Calcagno A, Gardiman M, Dal Moro F, Artibani, W. The novel hormone INSL3 is expressed in human testicular Leydig cell tumors: a clinical and immunohistochemical study. Plagiarism. (2011) 29:33-7. doi: 10.1016/j.urolonc.2008.10.015

36. Hampel U, Klonisch T, Sel S, Schulze U, Paulsen FP. Insulin-like factor 3 promotes wound healing at the ocular surface. Endocrinology. (2013) 154:2034-45. doi: 10.1210/en.2012-2201

37. Ross SA, Davis CD. The emerging role of microRNAs and nutrition in modulating health and disease. Annu. Rev. Nutr. (2014) 34:30536. doi: 10.1146/annurev-nutr-071813-105729

38. Ter Horst R, Jaeger M, Smeekens SP, Oosting M, Swertz MA, Li Y, et al. Host and environmental factors influencing individual human cytokine responses. Cell. (2016) 167:1111-24.e13. doi: 10.1016/j.cell.2016. 10.018

39. Sambarey A, Devaprasad A, Mohan A, Ahmed A, Nayak S, Swaminathan $\mathrm{S}$, et al. Unbiased identification of blood-based biomarkers for pulmonary tuberculosis by modeling and mining molecular interaction networks. EBioMedicine. (2017) 15:112-26. doi: 10.1016/j.ebiom.2016. 12.009
40. Rajan JV, Semitala FC, Mehta T, Seielstad M, Montalvo L, Andama A, et al. A novel, 5-transcript, whole-blood gene-expression signature for tuberculosis screening among people living with human immunodeficiency virus. Clin. Infect. Dis. (2019) 69:77-83. doi: 10.1093/cid/ciy835

41. Ranjbar S, Jasenosky LD, Chow N, Goldfeld AE. Regulation of Mycobacterium tuberculosis-dependent HIV-1 transcription reveals a new role for NFAT5 in the toll-like receptor pathway. PLoS Pathogens. (2012) 8:e1002620. doi: 10.1371/journal.ppat.1002620

42. Kroon EE, Coussens AK, Kinnear C, Orlova M, Möller M, Seeger A, et al. Neutrophils: innate effectors of TB resistance? Front. Immunol. (2018) 9:2637. doi: 10.3389/fimmu.2018.02637

43. Sutherland JS, Loxton AG, Haks MC, Kassa D, Ambrose L, Lee JS, et al. Differential gene expression of activating $\mathrm{Fc} \gamma$ receptor classifies active tuberculosis regardless of human immunodeficiency virus status or ethnicity. Clin. Microbiol. Infect. (2014) 20:O230-8. doi: 10.1111/1469-0691.12383

44. Kassa D, Ran L, Jager W, van den Broek T, Jacobi R, Mekonen M, et al. Discriminative expression of whole blood genes in HIV patients with latent and active TB in Ethiopia. Tuberculosis. (2016) 100:2531. doi: 10.1016/j.tube.2016.06.003

45. Gebremicael G, Kassa D, Quinten E, Alemayehu Y, Gebreegziaxier A, Belay Y, et al. Host gene expression kinetics during treatment of tuberculosis in HIVcoinfected individuals is independent of highly active antiretroviral therapy. J. Infect Dis. (2018) 218:1833-46. doi: 10.1093/infdis/jiy404

Conflict of Interest: The authors declare that the research was conducted in the absence of any commercial or financial relationships that could be construed as a potential conflict of interest.

Copyright (C) 2021 Kulkarni, Queiroz, Sangle, Kagal, Salvi, Gupta, Ellner, Kadam, Rolla, Andrade, Salgame and Mave. This is an open-access article distributed under the terms of the Creative Commons Attribution License (CC BY). The use, distribution or reproduction in other forums is permitted, provided the original author(s) and the copyright owner(s) are credited and that the original publication in this journal is cited, in accordance with accepted academic practice. No use, distribution or reproduction is permitted which does not comply with these terms. 